Article

\title{
Development of Folic Acid-Conjugated and Methylene Blue-Adsorbed Au@TNA Nanoparticles for Enhanced Photodynamic Therapy of Bladder Cancer Cells
}

\author{
Che-Wei Hsu ${ }^{1,+}{ }^{\dagger}$ Nai-Chi Cheng ${ }^{2,+}$, Mei-Yi Liao ${ }^{3, \dagger}{ }^{+}$Ting-Yu Cheng ${ }^{3}$ and Yi-Chun Chiu ${ }^{4,5,6, *}$ \\ 1 Division of Urology, Department of Surgery, Taipei City Hospital Zhongxiao Branch, Taipei 115, Taiwan; \\ DAX82@tpech.gov.tw \\ 2 Department of Applied Chemistry, National University of Kaohsiung, Kaohsiung 811, Taiwan; \\ ferrero565@gmail.com \\ 3 Department of Applied Chemistry, National Pingtung University, Pingtung 900, Taiwan; \\ myliao@mail.nptu.edu.tw (M.-Y.L.); t0922460558@gmail.com (T.-Y.C.) \\ 4 Division of Urology, Department of Surgery, Taipei City Hospital Heping Fuyou Branch, Taipei 100, Taiwan \\ 5 Department of Exercise and Health Sciences, University of Taipei, Taipei 100, Taiwan \\ 6 Department of Urology, School of Medicine, National Yang-Ming University, Taipei 112, Taiwan \\ * Correspondence: DAM15@tpech.gov.tw; Tel.: +886-2-23889595 (ext. \#7999) \\ + These authors contributed equally to this work.
}

Received: 11 May 2020; Accepted: 8 July 2020; Published: 10 July 2020

check for updates

\begin{abstract}
Photodynamic therapy (PDT) is a promising treatment for malignancy. However, the low molecular solubility of photosensitizers (PSs) with a low accumulation at borderline malignant potential lesions results in the tardy and ineffective management of recurrent urothelial carcinoma. Herein, we used tannic acid (TNA), a green precursor, to reduce $\mathrm{HAuCl}_{4}$ in order to generate $\mathrm{Au} @ \mathrm{TNA}$ core-shell nanoparticles. The photosensitizer methylene blue (MB) was subsequently adsorbed onto the surface of the Au@TNA nanoparticles, leading to the incorporation of a PS within the organic shell of the Au nanoparticle nanosupport, denoted as Au@TNA@MB nanoparticles (NPs). By modifying the surface of the Au@TNA@MB NPs with the ligand folate acid (FA) using $\mathrm{NH}_{2}-\mathrm{PEG}_{-} \mathrm{NH}_{2}$ as a linker, we demonstrated that the targeted delivery strategy achieved a high accumulation of PSs in cancer cells. The cell viability of T24 cells decreased to $87.1 \%, 57.1 \%$, and $26.6 \%$ upon treatment with 10 ppm $_{[\mathrm{Au}]}$ Au@TNA/MB NPs after 45 min, 2 h, and 4 h of incubation, respectively. We also applied the same targeted PDT treatment to normal urothelial SV-HUC-1 cells and observed minor phototoxicity, indicating that this safe photomedicine shows promise for applications aiming to achieve the local depletion of cancer sites without side effects.
\end{abstract}

Keywords: photodynamic therapy; bladder cancer; photosensitizers; Au@TNA nanoparticles; phototoxicity; photomedicine

\section{Introduction}

Bladder cancer is the 10th most common cancer worldwide, with an estimated 549,000 new cases and 200,000 deaths each year [1]. Nearly 70\% of bladder cancer cases are superficial or non-muscle-invasive bladder cancer (NMIBC) at initial presentation [2]. Initial treatment options include complete transurethral resection (TUR), followed by intravesical bacillus Calmette-Guérin (BCG) $[3,4]$. Nevertheless, 55\% of NMIBC patients develop recurrence with limited treatment options, and $20 \%$ progress to muscle-invasive bladder cancer (MIBC) within 5 years [5]. The condition of those patients will exacerbate rapidly if left untreated, and the mortality within 2 years is 85\% [6]. 
A multidisciplinary therapeutic approach tailored to individual patients, including surgery, systemic chemotherapy, and radiotherapy, is often required to improve survival; however, these treatments may cause severe adverse effects and affect the quality of life. These management steps will affect the daily urinary storage and voiding function of the bladder, which is crucial for allowing patients to eliminate waste from the body, regulate their blood pressure, and control levels of electrolytes. Focal surgical intervention combined with minimally invasive therapeutic techniques for bladder cancer would alleviate the adverse effects and inconvenience caused by the long-term course of treatment [7].

Photodynamic therapy (PDT) is a minimally invasive therapeutic procedure used for malignant cells that involves the administration of a photosensitizer (PS) followed by irradiation at a wavelength corresponding to the absorbance band of the PS and a series of events leading to direct tumor cell death [8]. The clinical use of many photosensitizers has been hampered by their nonspecific damage to normal tissues, environmental degradation or hydrophobicity, and poor cellular uptake [9]. The exact reasons for the preferential accumulation of PSs in cancer tissue have not been clearly elucidated. Some of the hypotheses include leaky tumor vasculature, reduced lymphatic drainage, a low interstitial $\mathrm{pH}$, and a high number of low-density lipoprotein (LDL) receptors [10]. The most widely discussed photosensitizers are porfimer sodium (Photofrin) and hexaminolevulinate (HAL). The response rate to conventional therapy from combined series is $66 \%$ in patients with carcinoma in situ (CIS) of the bladder refractory [11]. Most lipophilic photosensitizers associated with PDT (e.g., Photofrin) localize to the mitochondria and induce apoptosis through mitochondrial disruption, the release of cytochrome c, and activation of the intrinsic pathway of apoptosis [12]. Because of their lipophilicity, they tend to enter both normal and neoplastic cells, leading to collateral damage [13]. The dermal sensitivity and bladder toxicity of PSs not only limit the safe dose, but also induce significant side effects, such as skin photosensitivity, bladder contracture, fibrosis, irritability, and even a loss of bladder capacity. A new targeted PS delivery approach has emerged from the PDT method; this approach combines a very hydrophilic PS to minimize nonspecific accumulation due to the natural hydrophobicity of normal urothelial cells and a PS conjugated to monoclonal antibodies specifically selected for proteins overexpressed on the surface of cancer cells [10]. Although selectively targeting antibody-conjugated photosensitizers to tumors can protect normal urothelium cells and avert adverse events, the amount of drug delivered remains lower than those obtained using designed carriers based on polymerases, micelles, and high surface-to-volume ratio nanoparticles (NPs).

Noncytotoxic materials such as gold, iron oxide, and silica NPs are becoming promising drug carrier platforms for biomedical applications [14,15]. Gold NPs (AuNPs) are emerging as multifunctional agents for cancer therapy and are being investigated as drug carriers, photothermal agents, and radiosensitizers owing to their high biocompatibility and well-defined optical properties [16,17]. Because potential sensitivity to the oxygen environment may cause unpredictable cytotoxicity, congeneric copper $(\mathrm{Cu})$ [18] and silver (Ag) [19] NPs, or even inert platinum (Pt)-based particles [20], are not applicable drug carriers. Methylene blue (MB) is a phenothiazinium photosensitizer due to its high quantum yield of ${ }^{1} \mathrm{O}_{2}$ generation under excitation in the therapeutic window (600-900 nm) and has an excellent membrane-permeable ability [21,22]. MB-encapsulated NP-based phototherapy is very encouraging [23, 24] as a photomedicine platform [25] because MB can undergo near-infrared (NIR) excitation to reach deeper depths of tissue. However, the self-aggregation of MB in tumor sites leads to a lower quantum yield of singlet oxygen production, thus limiting its practical application in clinical settings [22].

To deliver massive therapeutic molecules, the application and synthesis of gold NPs have been discussed in many previous works [26,27]; such approaches may require organic materials decorated on the surface of the NPs to prevent particle aggregation and facilitate further bioconjugation [28]. However, preparing a surface passivation layer and carrying PS [29,30] requires postsynthesis reactions and utilizes extra chemical reagents. It is possible that the reaction chemicals residing in the NP carrier may cause unknown toxicity when living cells meet the designed Au-based NPs. Herein, we developed a tannic acid (TNA)-assisted reduction of $\mathrm{HAuCl}_{4}$ to form $\mathrm{Au} @ \mathrm{TNA}$ core-shell NPs. The synthesis of gold nanoparticles by the assisted reduction of polyphenols is a simple, low-cost, 
long-term stable, eco-friendly, and green chemistry approach [31]. Huang and co-workers developed an in-situ reduction process of $\mathrm{HAuCl}_{4}$ with tannic acid for the direct deposition of $\mathrm{Au}$ atoms onto filter paper [32]. The TNA capping layer was capable of the monomer-based adsorption of MB molecules within the TNA organic shell, leading to a high yield of ${ }^{1} \mathrm{O}_{2}$ molecules. The TNA/MB passivation layer readily protected the Au NPs against aggregation in PBS solution over 7 days. MTT assays confirmed the low cytotoxicity of the Au@TNA and Au@TNA@MB NPs. Upon conjugation with folic acid molecules [33,34], Au@TNA/MB exhibited a better selectivity to bind the folate receptor on HeLa cervical cancer cells and T24 bladder cancer cells. Therefore, targeted PDT treatment could produce a large number of reactive oxygen species (ROS) to efficiently damage cancer cells upon $650 \mathrm{~nm}$ irradiation, while neither dark toxicity nor a nontoxic PDT effect on the SV-HUC-1 normal bladder cell line was observed. Our approach used a targeted PS to deliver PDT and indicates that the promising next-generation development of selective PDT could be combined with photoimmunotherapy [10] for bladder tumor treatment.

\section{Materials and Methods}

\subsection{Materials}

Tannic acid (TNA), the photosensitizer methylene blue (MB), thiazolyl blue tetrazolium bromide (MTT), and $N$-hydroxysuccinimide (NHS) were obtained from Alfa Aesar. Imidazole $(98 \%)$, $\mathrm{N}, \mathrm{N}$-dimethyl-4-nitrosoaniline (RNO), folic acid (FA), and ethyl (dimethylaminopropyl) carbodiimide (EDC) were purchased from Sigma-Aldrich (St. Louis, MO, USA). Hydrogen tetrachloroaurate(III) trihydrate $\left(\mathrm{HAuCl}_{4} \cdot 3 \mathrm{H}_{2} \mathrm{O}, 99.9 \%\right)$ was obtained from Bio-Tech. Dimethyl sulfoxide (DMSO) was purchased from Scharlau (Sentmenat, Spain). $\mathrm{NH}_{2}-\mathrm{PEG}_{3500}-\mathrm{NH}_{2}$ and 9,10-anthracenediyl-bis (methylene) dimalonic acid (ABDA) were purchased from JenKem Technology and FLUKA, respectively. These chemicals were used as received. Deionized water (DI water) was used as a solvent to dissolve the hydrophilic chemicals.

\subsection{Methods}

\subsubsection{Preparation of $A u @ M B ~ N P s$}

$\mathrm{HAuCl}_{4}$ solution $(4.5 \mathrm{~mL}, 1.1 \mathrm{mM})$ was mixed with fresh TNA solution $(0.5 \mathrm{~mL}, 2.5 \mathrm{mM})$ at room temperature. After $1 \mathrm{~h}$ of reaction, the resulting Au@TNA nanoparticles were collected and purified with DI water by a centrifugation/resuspension process more than three times. The final product was dispersed in $5 \mathrm{~mL}$ DI water.

MB powder was dissolved in DI water to prepare a $5 \mathrm{mM}$ mother solution. Afterwards, $25 \mu \mathrm{L}$ MB (5 mM) was added to the above $5 \mathrm{~mL}$ colloidal solution of Au@TNA nanoparticles. The mixture solution was aged for $24 \mathrm{~h}$. After a purification treatment with centrifugation at 10,000× $\mathrm{g} \mathrm{rpm}$ and redispersion with DI water more than three times to remove the excess MB molecules, Au@TNA@MB nanoparticles were collected. The amount of MB adsorbed onto the surface of Au@TNA nanoparticles was calculated from the difference between the initial optical density of $\mathrm{MB}$ and each supernatant collected from centrifugation.

To measure the amount of MB adsorbed onto the Au@TNA nanoparticles, the absorption peak at $660 \mathrm{~nm}$ was recorded using a UV-visible spectrometer. The difference between the initial amount of MB and the residue in the supernatant of the Au@TNA and MB mixture solution was calculated, resulting in a $70 \%$ reduction ratio. Based on the absorption measurement, $3.4 \mu \mathrm{M} \mathrm{MB}$ was attached to 40 ppm Au@TNA nanoparticles, which corresponded to $27.5 \mathrm{mg}$ [MB] $/ \mathrm{g}_{[\mathrm{Au}]}$.

\subsubsection{Singlet Oxygen Yielded by Au@TNA@MB NPs and MB}

A total of $200 \mathrm{uL}$ Au@TNA@MB (25 ppm [Au] $)$ was mixed with $10 \mathrm{uL}$ RNO $(1 \mathrm{mM})$ and $10 \mu \mathrm{L}$ imidazole $(1 \mathrm{mM})$ solution, and then exposed to $650 \mathrm{~nm}$ laser light. The absorption band at $440 \mathrm{~nm}$ 
was recorded as a function of the irradiation time. The same experiment was carried out for assessing the yield of ${ }^{1} \mathrm{O}_{2}$ by $\mathrm{Au} @ \mathrm{TNA} @ \mathrm{MB}\left(25 \mathrm{ppm}_{[\mathrm{Au}]}\right)$ and MB solution $(0.024 \mathrm{mM})$.

ABDA was used as another ${ }^{1} \mathrm{O}_{2}$ indicator. A total of $200 \mu \mathrm{L}$ Au@TNA@MB NPs $(25$ ppm $[\mathrm{Au}]$ was mixed with $10 \mu \mathrm{L}$ ABDA solution $(5 \mathrm{mM})$, followed by the same irradiation process. The absorption change at $400 \mathrm{~nm}$ of all samples was recorded.

\subsubsection{Synthesis of FA-Conjugated Au@TNA@MB NPs}

Two hundred microliters of Au@TNA/MB NP solution (200 ppm [Au] $_{\text {( }}$ ) was mixed with $200 \mu \mathrm{L}$ of $\mathrm{NH}_{2}$-PEG-NH $\mathrm{NH}_{2}$ polymer solution $(2.8 \mathrm{mg} / \mathrm{mL})$ at $4{ }^{\circ} \mathrm{C}$ for one hour, referred to as solution A. Then, $7 \mathrm{mg} / \mathrm{mL} N$-(3-dimethyl aminopropyl)- $N^{\prime}$-ethyl carbodiimide hydrochloride (EDC) and $7 \mathrm{mg} / \mathrm{mL}$ $\mathrm{N}$-hydroxysuccinimide (NHS) were dissolved in $0.85 \mathrm{~mL}$ of FA solution $(0.025)$ to activate the carboxylate groups, referred to as solution B. Solutions A and B were subsequently reacted for another $2 \mathrm{~h}$ to form FA-conjugated Au@TNA@MB NPs, where the primary amine groups of the $\mathrm{NH}_{2}-\mathrm{PEG}-\mathrm{NH}_{2}$ polymer were used to link the Au@TNA@MB NPs and FA. Centrifugation at 10,000× g rpm and redispersion with DI water more than three times were implemented to purify FA-conjugated Au@TNA@MB NPs.

\subsubsection{Cytotoxicity of Au@TNA/MB NPs against Bladder Cancer Cells}

A cell toxicity test using a series of as-prepared Au@TNA NPs, Au@TNA@MB NPs, FA-conjugated Au@TNA@MB NPs, Au@TNA@MB NPs plus 660 nm light, and FA-conjugated Au@TNA@MB NPs plus $660 \mathrm{~nm}$ light was performed on HeLa cells and T24 cells. HeLa cells, T24 cells, and SV-HUC-1 cells were precultured in DMEM-HG culture medium, McCoy's 5A medium, and Ham's F-12K (Kaighn's) medium, respectively, for $24 \mathrm{~h}$ at $37^{\circ} \mathrm{C}$ under $5 \% \mathrm{CO}_{2}$ culture conditions. We tested the cell viability of different cells with Au-based nanoparticles at a density of 8000 cells per well in a 96-well plate. After 1 day of seeding, we dispersed these Au-based nanomaterials in culture media and then added $100 \mu \mathrm{L}\left(0-200 \mathrm{ppm}_{[\mathrm{Au}]}\right)$ to each well to replace the original culture medium. After $45 \mathrm{~min}, 2 \mathrm{~h}$, and $4 \mathrm{~h}$ of coculture, the particle-including medium was removed and then replaced by $100 \mu \mathrm{L}$ of fresh culture medium. These experimental groups were irradiated with a $660 \mathrm{~nm}$ laser $\left(125 \mathrm{~mW} / \mathrm{cm}^{2}\right)$ for $8 \mathrm{~min}$. The PDT-treated cells or dark-treated (without light) groups were then kept in the incubator for another day. The cell viability was determined by utilizing the MTT assay to measure the ratio difference between the treated groups and the cell-only group (without treatment in the form of particles and laser light).

2.2.5. Cellular Response to Membrane Destruction and Reactive Oxygen Species Detection with Trypan Blue and DCFH-DA

To assess whether the PDT resulted in killing efficiency, living cells treated with Au@TNA@MB NPs plus 660 nm light and FA-conjugated Au@TNA@MB NPs were stained with trypan blue to test the cell viability and with DCFH-DA to analyze the ROS level.

We incubated HeLa cells with $100 \mu \mathrm{L}$ of the vital stain trypan blue $(5 \mathrm{mM})$ for $30 \mathrm{~min}$ after performing PDT treatment (Au@TNA@MB (10 ppm $[\mathrm{Au}]))$. PBS solution was added to replace the original staining solution to remove the excess trypan blue. After another $4 \mathrm{~h}$ of incubation, a microscope was used to visualize the color pattern of the HeLa cells.

Ten microliters of DCFH-DA stock solution $(1 \mathrm{mM})$ was mixed with $100 \mu \mathrm{L}$ of PBS solution for the following experiment. One milliliter of T24 cells $\left(10,000\right.$ cells/mL) was cultured for $24 \mathrm{~h}$ at $37^{\circ} \mathrm{C}$ under $5 \% \mathrm{CO}_{2}$ on a gelatin-coated microscope slide. The medium was removed and replaced with $200 \mu \mathrm{L}$ of culture medium, including $20 \mu \mathrm{L}$ of diluted DCFH-DA solution. After an incubation time of $30 \mathrm{~min}$, we used PBS solution to remove the excess DCFH-DA, and $250 \mu \mathrm{L}$ of Au-based nanomaterials was then loaded into the DCFH-DA stained cells for $4 \mathrm{~h}$. The excess Au-based nanomaterials were removed and the PDT treatment was applied (660 $\mathrm{nm}$ laser irradiation at $125 \mathrm{~mW} / \mathrm{cm}^{2}$ for $8 \mathrm{~min}$ ). The ROS generation in cell images was investigated by using an Olympus microscope. 


\subsection{Characterization}

Transmission electron microscopy (TEM) images were taken by using an H-7500 instrument (HITACHI, Tokyo Japan). High-resolution TEM (HR-TEM) images were recorded by a JEM-2100F (JEOL Ltd., Tokyo, Japan). Powder X-ray diffraction (XRD) analysis was carried out on a Rigaku D/MAX2500 instrument. Ultraviolet-visible spectra were recorded using a V-630 spectroscope (JASCO, Tokyo, Japan). X-ray photoelectron spectroscopy (XPS) analysis was performed on an Ulvac-Phi 5000 Versaprobe instrument (Thermo-VG Scientific, West Sussex, UK). Micro-Raman spectroscopy was performed with a $785 \mathrm{~nm}$ laser (DPSSL Driver II, $3.4 \mathrm{~mW})$, an He-Ne laser at $632.8 \mathrm{~nm}(20 \mathrm{~mW})$, and an MRS-iHR320 modular Raman system, which were integrated into an Olympus BX53 microscope for cellular examinations. The microscope was incorporated with a 20X (N.A. $=0.5)$ objective lens for SERS sample measurement and combined with Olympus bandpass filter cubes (U-FGW (575-800 nm) and U-FBWA (510-550 nm)) to obtain the red and green emission, respectively.

\section{Results and Discussion}

\subsection{Characterization of $A u @ T N A / M B N P s$}

Figure 1a shows a TEM image of Au@TNA NPs prepared by a one-pot reaction of $\mathrm{HAuCl}_{4}$ and TNA solutions. These particles consisted of spherical and triangular shapes with an average particle size of $26.43 \pm 3.7 \mathrm{~nm}$ (Figure S1). Figure 1b,c shows HR-TEM images of individual Au@TNA NPs with a highly crystalline structure and spherical and triangular shapes, respectively. The crystal lattice space of the periodic packing fringes was $2.35 \AA$, which was consistent with the (111) diffraction corresponding to face-centered cubic-structured gold. Notably, the spherical gold NP contained three-fold symmetry projections, suggesting the formation of an icosahedral building block structure [35]. Upon the adsorption of methylene blue, the size $(26.88 \pm 4.6 \mathrm{~nm})$ and shape of the Au@TNA@MB NPs were almost the same as those of the Au@TNA NPs according to the TEM images (Figure 1d). The XRD pattern (Figure 1e) confirmed that the reflection peaks of the Au@TNA@MB NPs corresponded to the face-centered cubic pattern of gold crystallite. Figure $1 \mathrm{f}$ shows that the peak positions of $\mathrm{Au} 4 \mathrm{f}_{5 / 2}$ at $84.0 \mathrm{eV}$ and $\mathrm{Au} 4 \mathrm{f}_{7 / 2}$ at $87.6 \mathrm{eV}$ were consistent before and after loading MB. The lack of an upshift change at the Au $4 \mathrm{f}$ orbital position [36] indicated that the protective TNA interlayer separated sulfur atoms in MB and Au NPs.

Ultraviolet-visible (UV) and fluorescence spectra of Au@TNA and Au@MB NPs from wavelengths between 200 and $900 \mathrm{~nm}$ were recorded and are shown in Figure 2a. The absorption peak of the localized surface plasmon resonance (LSPR) of Au@TNA NPs shifted from 528 to 546 nm after the loading of MB molecules. This primary peak moved to a longer wavelength, which could be attributed to the increase in the refractive index after the decoration of a large amount of MB over the surface of Au@TNA NPs. Nearly 70\% of MB was adsorbed onto the surface of Au@TNA NPs (Figure S2). Accordingly, we observed that the solution color of Au@TNA NPs changed to a blue-like color for the Au@TNA@MB NP solution (Figure S3). When the Au-based colloids were added to PBS solution, the resulting Au@TNA@MB NP solution showed a high stability against aggregation after 7 days of aging (Figure S3b). In addition, we observed that the maximum absorption peak of MB adsorbed onto the surface of Au@TNA NPs was located at 664 nm, which was slightly redshifted compared with the $662 \mathrm{~nm}$ absorption peak of the pure MB solution (Figure 2a). Notably, the absorption band positions of Au@TNA NP and MB were overlapping at 600-650 nm. Additional simulations and experiments are required to examine the potential formation of J- or H-aggregation adsorption [37] of MB molecules on the surface of TNA-decorated Au NPs via hydrogen bonds and/or $\pi-\pi$ stacking interactions. Strong fluorescence appeared and negligible surface-enhanced Raman scattering peaks were obtained in the Raman spectrum under laser excitation at $633 \mathrm{~nm}$ (Figure 2b). The appearance of fluorescence interference can be attributed to the slight release of MB (about 2.4\%) from the Au@TNA@MB NPs (Figure S4). To eliminate the typical fluorescence interference in the SERS spectra from the 633-excited MB molecules, we utilized a $785 \mathrm{~nm}$ Raman system to analyze the same solution. The SERS effect of 
Au@TNA@MB NPs is very weak at $785 \mathrm{~nm}$. These opposite optical phenomena of high fluorescence and low SERS confirmed the existence of the TNA organic shell as a spacer, preventing the close adsorption of MB on the surface of Au NPs.
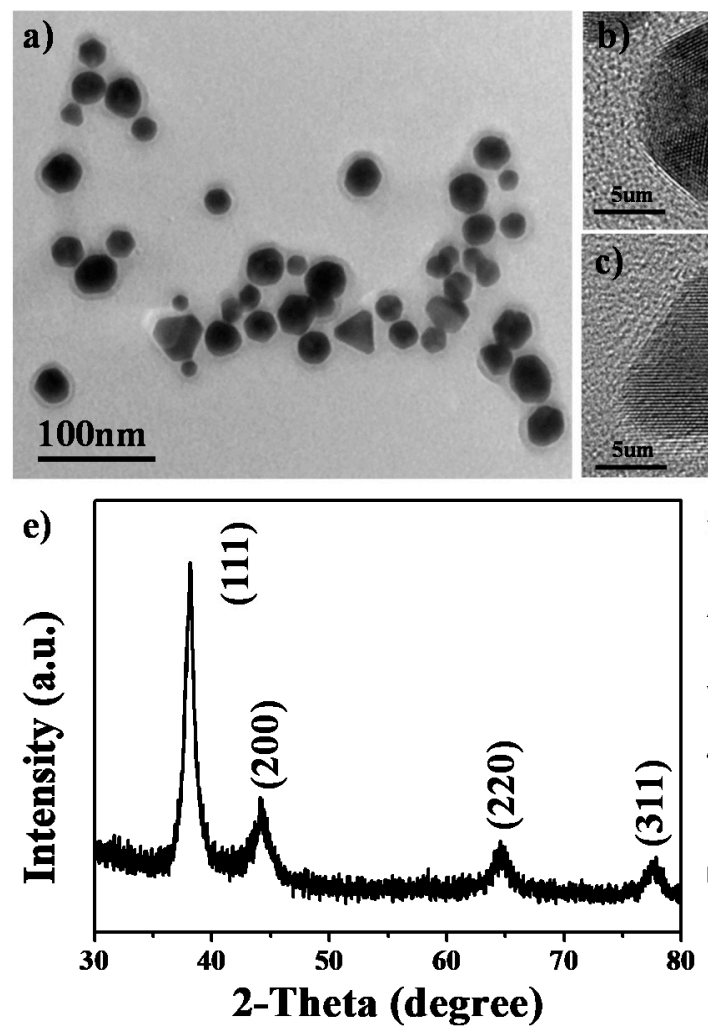
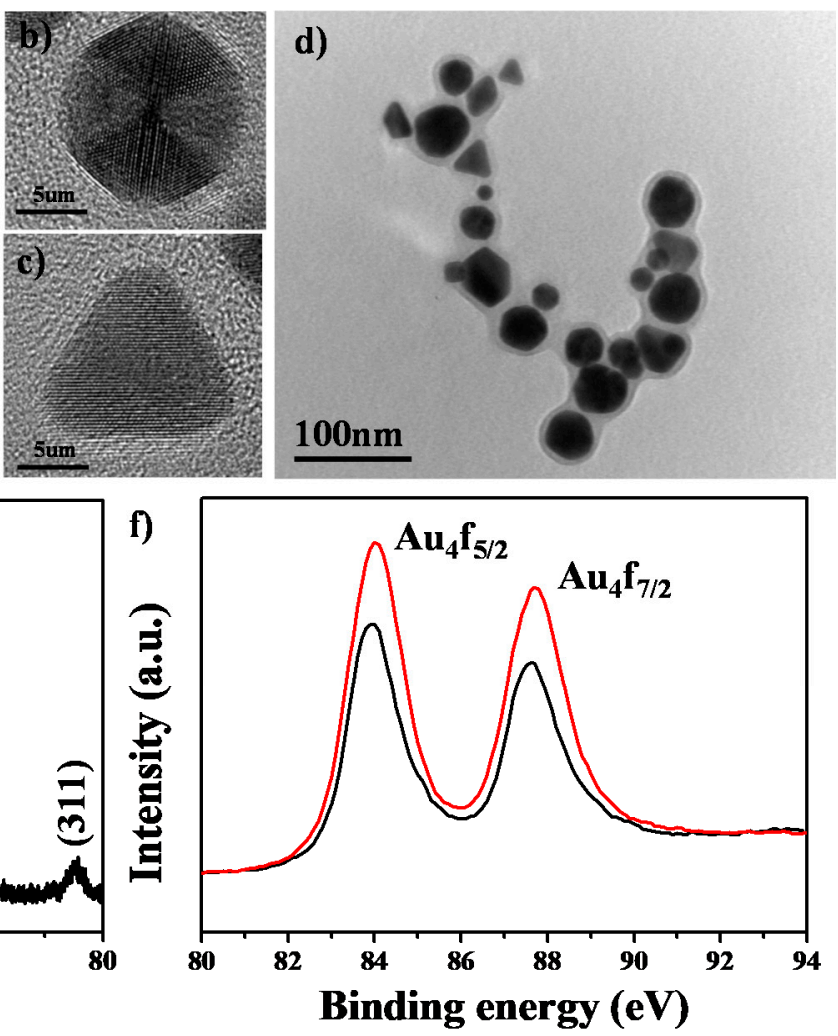

Figure 1. (a) Transmission electron microscopy (TEM) image and (b,c) High-resolution TEM (HR-TEM) images of Au@TNA nanoparticles (NPs). (d) TEM image of Au@TNA@MB NPs. (e) X-ray diffraction (XRD) pattern of Au@TNA@MB NPs. (f) X-ray photoelectron spectroscopy (XPS) gold (Au) 4f orbitals of Au@TNA (red) and Au@TNA@MB (black) NPs.
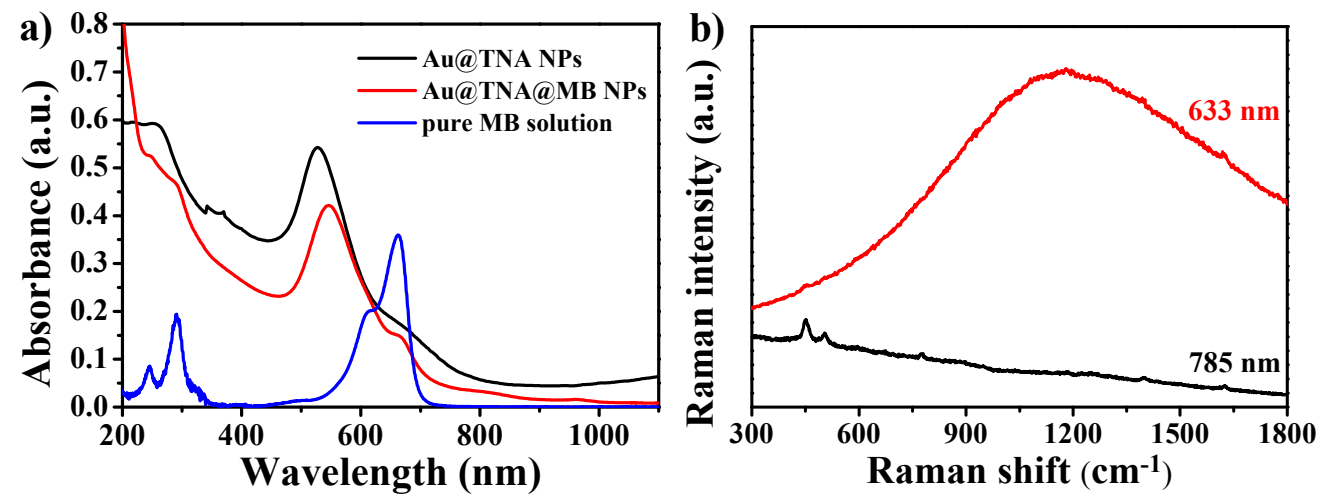

Figure 2. (a) UV-visible and (b) fluorescence spectra of Au@TNA NPs, Au@TNA@MB NPs, and methylene blue (MB) molecules. (b) SERS measurement of Au@TNA@MB NPs at 633 and 785 nm.

Next, we detected the production of ${ }^{1} \mathrm{O}_{2}$ from Au@TNA@MB NPs (350 ppm $[\mathrm{Au}]$ ) under irradiation with a $660 \mathrm{~nm}$ laser. We used a chemical mixture of imidazole and RNO as an indicator to detect the ${ }^{1} \mathrm{O}_{2}$ generation of Au@TNA/MB NPs. After reacting Au@TNA/MB NPs and the indicator solution under a $660 \mathrm{~nm}$ NIR laser $\left(125 \mathrm{~mW} / \mathrm{cm}^{2}\right)$, a continuous decrease in the absorption peak at $440 \mathrm{~nm}$ was observed from 2 to $16 \mathrm{~min}$ (Figure 3a). The relative decreases observed for MB alone, Au@TNA@MB NPs, and Au@TNA NPs were 83\%, 42.15\%, and 3.2\%, respectively (Figure 3b). A similar decreasing 
trend was observed by using an ABDA indicator. Indeed, Au@TNA@MB NPs appeared to have a lower ${ }^{1} \mathrm{O}_{2}$ efficiency when compared with the MB-alone group (Figure S5). When the excited-state MB molecule was transformed and adsorbed on the surface of the Au NPs, the energy quenching process gently decreased ${ }^{1} \mathrm{O}_{2}$ generation.
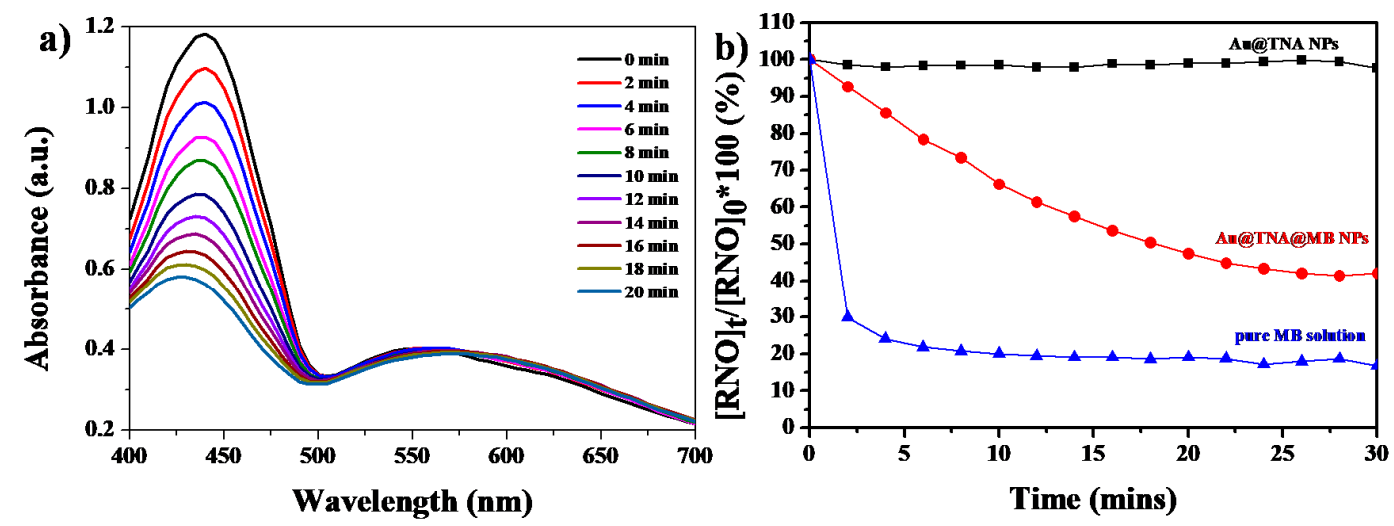

c) $0 \mathrm{~min}$ $2 \mathrm{~min}$ $4 \mathrm{~min}$

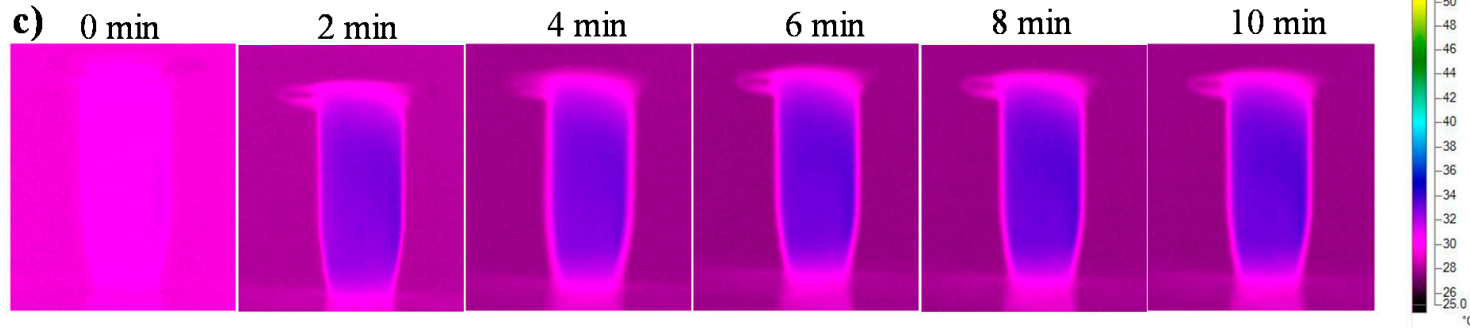
$8 \mathrm{~min}$ $10 \mathrm{~min}$

Figure 3. UV-visible spectra (a) and relative intensity at $440 \mathrm{~nm}(\mathbf{b})$ of the $N, N$-dimethyl-4-nitrosoaniline (RNO)/imidazole indicator for measuring single oxygen generation from Au@TNA@MB NPs as a function of the irradiation time at $650 \mathrm{~nm}$. Pure MB and Au@TNA are presented as control groups. (c) Thermographic images mapping the temperature increase of 25 ppm $[\mathrm{Au}]$ Au@TNA@MB NPs at 650 $\mathrm{nm}\left(125 \mathrm{~mW} / \mathrm{cm}^{2}\right)$.

In addition, the Au@TNA@MB NPs exhibited a consistent heating temperature below $38{ }^{\circ} \mathrm{C}$ after $10 \mathrm{~min}$ of irradiation at $660 \mathrm{~nm}$ (Figure 3c). Compared to the MB-alone group (Figure S6), the absorption of Au@TNA@MB NPs showed a negligible change at 664 nm (Figure 3a), which indicated a better photostability of Au@TNA@MB than MB alone. Although pure MB has a higher quantum yield of ${ }^{1} \mathrm{O}_{2}$ molecules, it is less applicable than Au@TNA/MB NPs for clinical use because of its unstable properties under prolonged excitation or upon accumulation in tumor sites [22]. On the other hand, the thermographic mapping results show the lack of a significant temperature increase after 8 min of excitation in Figure 3c. This temperature dependence of the Au@TNA/MB NPs (25 ppm) as a function of the laser irradiation time demonstrated a low potential risk of thermal ablation to living cells during $650 \mathrm{~nm}$ laser irradiation in the subsequent in vitro studies.

\subsection{Cytotoxicity of $A u @ T N A / M B N P s$ in PDT}

To validate the biocompatibility of Au@TNA/MB NPs, HeLa cells, representing a human cell line derived from a cervical cancer line, were cocultured with solid NPs for $24 \mathrm{~h}$ and analyzed by using the MTT assay, in order to evaluate the cell viability (Figure 4a). HeLa cells are one of the most typical cell models used for the preliminary study of PDT treatment models [38-40]. As shown in Figure 4a, no dark toxicity towards HeLa cells without light exposure was displayed by 0-200 ppm [Au] Au@TNA@MB NPs. As expected, we observed that 50-200 ppm[Au] Au@TNA@MB NPs effectively reduced the cell viability of HeLa cells to nearly $10 \%$ under $660 \mathrm{~nm}$ light irradiation. At low sample doses between 1 and 10 ppm $_{[\mathrm{Au}]}$, the PDT treatment was mildly harmful, with up to 65\% photoinduced 
cytotoxicity. In contrast, the treatment of Au@TNA NPs plus light was PDT inactive in causing injury to HeLa cells.

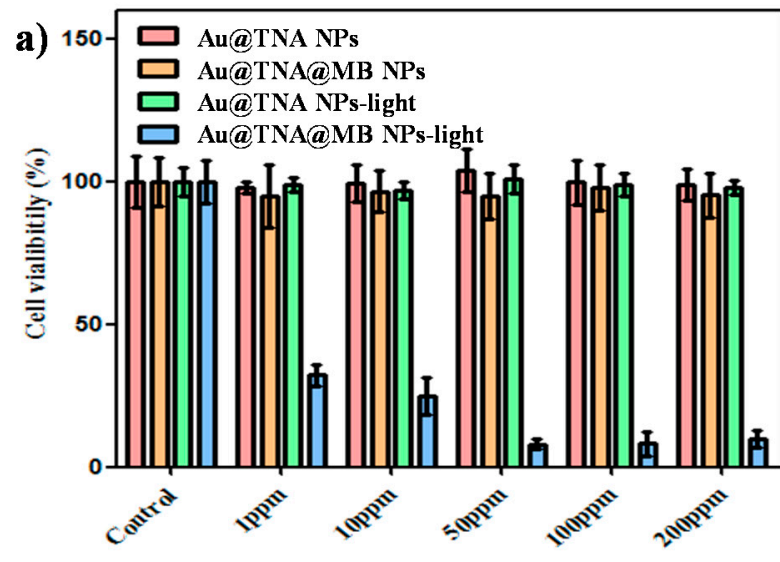

$4 \mathrm{hrs}$

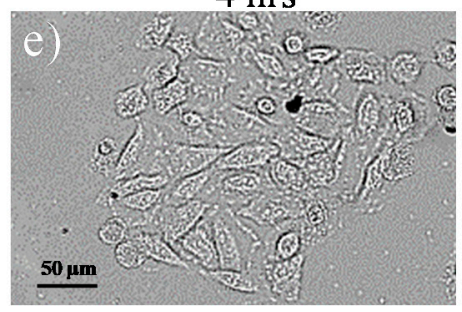

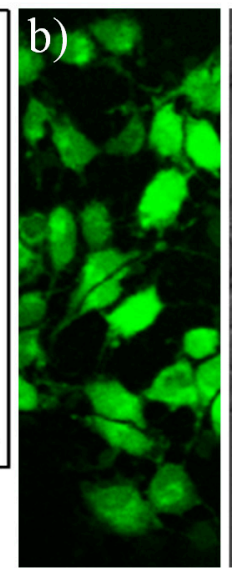

$6 \mathrm{hrs}$

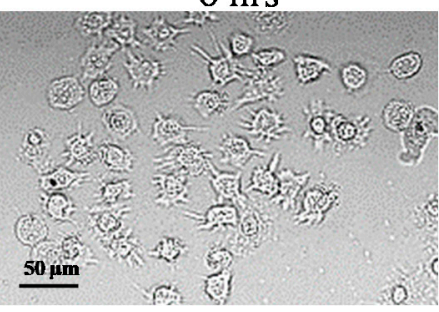

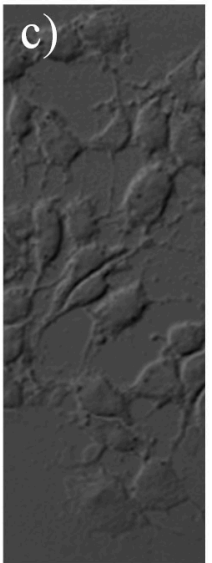

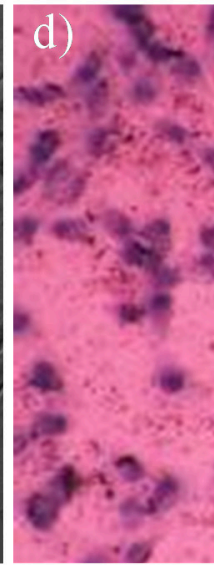

$24 \mathrm{hrs}$

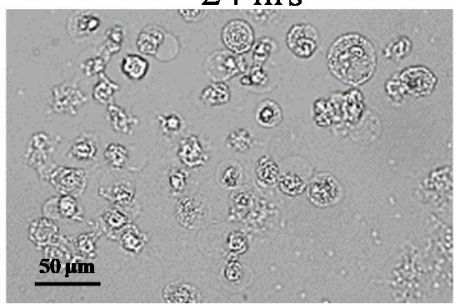

Figure 4. (a) Thiazolyl blue tetrazolium bromide (MTT) assay of HeLa cells cotreated with particles for 24 h: Au@TNA, Au@TNA@MB, Au@TNA plus650 nm light, and Au@TNA@MB plus 650 nm light. (b) 2',7'-dichlorofluorescin diacetate (DCFHDA)-stained HeLa cells, (c) bright field image of HeLa cells, and (d) trypan blue-stained HeLa cells. The cells were stained after $4 \mathrm{~h}$ of treatment with Au@TNA@MB and then exposed to 650 nm light. (e) Bright field images of HeLa cell apoptosis after pretreatment with 10 ppm $[\mathrm{Au}] \mathrm{Au} @ \mathrm{TNA} @ \mathrm{MB}(4 \mathrm{~h})$ plus $650 \mathrm{~nm}$ light. The $650 \mathrm{~nm}$ laser power density was $125 \mathrm{~mW} / \mathrm{cm}^{2}$.

The Au@TNA@MB NP-mediated PDT was investigated by using a nonfluorescent compound $\left(2^{\prime}, 7^{\prime}\right.$-dichlorofluorescin diacetate, DCFDA $)$ to produce a green fluorescent molecule $\left(2^{\prime}, 7^{\prime}\right.$-dichlor ofluorescein, DCF) when the cells received intracellular reactive oxygen species (ROS). After incubation with Au@TNA@MB NPs $\left(10 \mathrm{ppm}_{[\mathrm{Au}]}\right)$ for $4 \mathrm{~h}$ at $37^{\circ} \mathrm{C}$ under a dark condition, particle-treated HeLa cells were exposed to $650 \mathrm{~nm}$ laser light $\left(125 \mathrm{~mW} / \mathrm{cm}^{2}\right)$ for $8 \mathrm{~min}$. Consequently, the green fluorescence of DCF was clearly observed in Figure $4 \mathrm{~b}$ for Au@MB NP-treated HeLa cells after light irradiation, being well-matched with the cell location in the bright field image (Figure 4c), indicating the remotely controlled intracellular release of toxic ROS species. In addition, a trypan blue staining method was further used to examine the cell viability of irradiated HeLa cells after $4 \mathrm{~h}$ of PDT treatment (Figure 4d). The PDT-treated cells presented a blue color due to the diffusion of trypan blue across the membrane and into the cytoplasm, indicating that the large amount of ROS production within HeLa cells causes cell membrane damage and then triggers cell death. In contrast, the Au@TNA@MB NP w/o light group presented color-free cells, corresponding to a complete cell membrane structure, and thus resisted the inward diffusion of trypan blue. The time-dependent imaging results of PDT treatment are shown in Figure 4e. Consistently, the PDT-treated HeLa cells showed a morphological change: the cell membranes were gradually converted into shriveled and spiked structures from 4 to $6 \mathrm{~h}$, and membrane blebbing appeared at $24 \mathrm{~h}$, possibly through an apoptosis pathway [41]. The apoptotic membrane protrusions of the HeLa cells became round and started to detach from the culture dishes after $24 \mathrm{~h}$ of culture. Indeed, the cell volume contraction of PDT-treated HeLa cells from 4 to $24 \mathrm{~h}$, which was at least two-fold, suggests apoptosis pathways [42]. 
Again, we investigated the PDT killing effect on the T24 human bladder cancer cell line

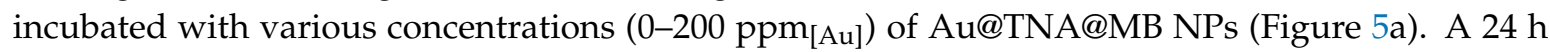
coincubation followed by PDT was performed. No cytotoxicity against T24 cells was observed when the culture conditions involved Au@TNA NPs, Au@TNA NPs plus light, and Au@TNA@MB NPs alone. The PDT-treated T24 cell group exhibited a remarkably low cell viability at 50-200ppm [Au] and an at least $70 \%$ cell death ratio at $1-10 \mathrm{ppm}_{[\mathrm{Au}]}$. Figure $5 \mathrm{~b}$ presents the results of time-dependent and dose-dependent coincubation conditions, which were used to assess the optimal PDT treatment for eliminating bladder cancers at a low drug dose within an ideal reaction time. After 45 min of incubation, 0.1-10 ppm Au@TNA/MB NPs did not have a significant phototoxic effect on T24 cells. There was only a mild killing rate of $42.8 \%$ at 10 ppm $_{[\mathrm{Au}]} \mathrm{Au} @ \mathrm{TNA} / \mathrm{MB}$ NPs for the PDT-treated T24 cells when the incubation time was prolonged to $2 \mathrm{~h}$. At $4 \mathrm{~h}$ of incubation, the T24 cell viability with 1, 5, and 10 ppm $_{[\mathrm{Au}]} \mathrm{Au} @ \mathrm{TNA} / \mathrm{MB}$ NPs decreased in a dose-dependent manner to 78.7\%, 59.6\%, and $26.6 \%$, respectively.
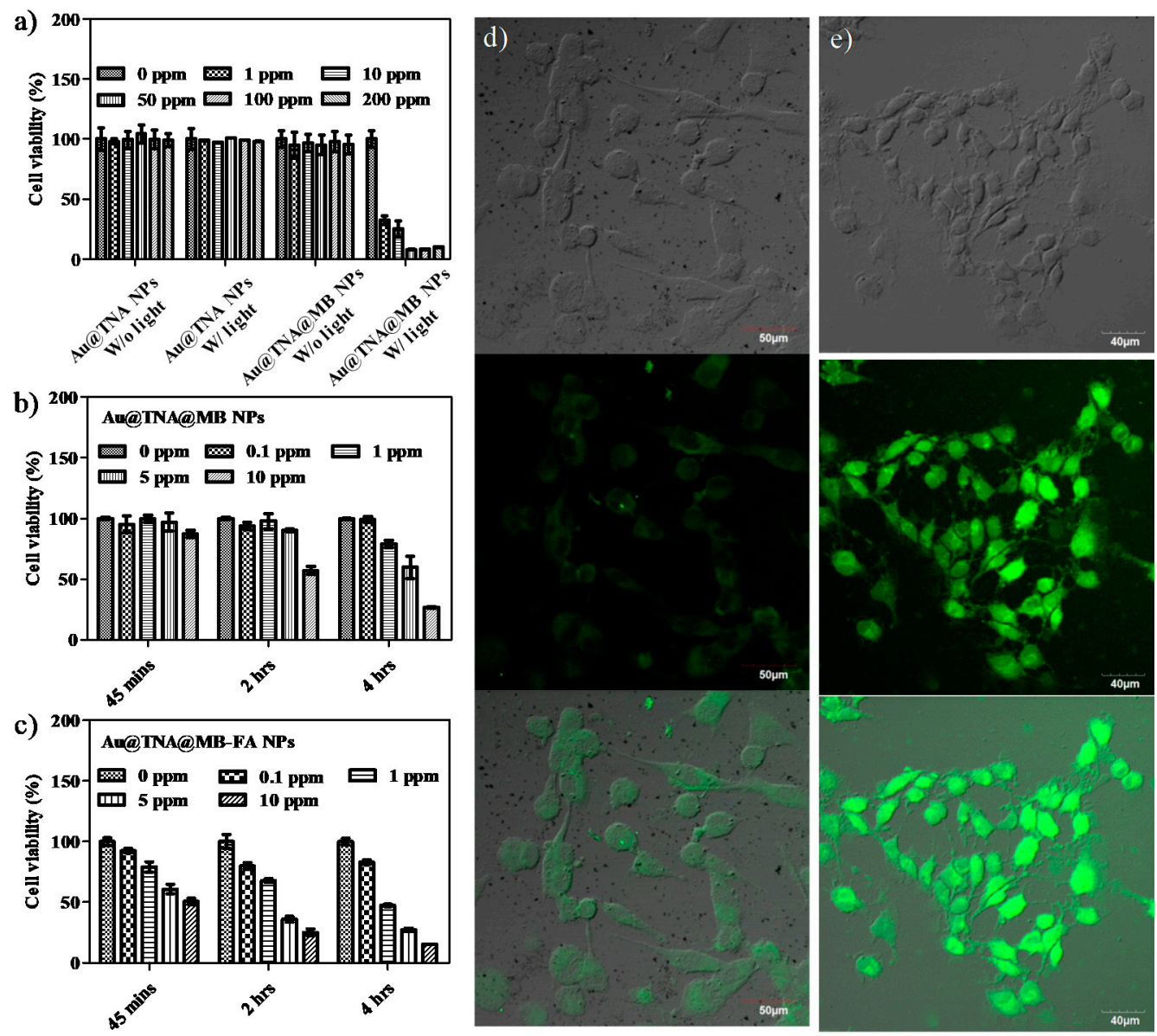

Figure 5. MTT assays of (a) T24 cells cotreated with particles for 24 h: Au@TNA, Au@TNA@MB, Au@TNA plus 650 nm light, and Au@TNA@MB plus 650 nm light. T24 cells cotreated with (b) Au@TNA@MB NPs and (c) folic acid (FA)-conjugated Au@TNA@MB NPs for 0.75-4 h upon excitation at $650 \mathrm{~nm}$. Photodynamic therapy (PDT) treatment of T24 cells stained with DCFH-DA dye by using (d) Au@TNA@MB NPs and (e) FA-conjugated Au@TNA@MB NPs. Top: bright field image, middle: fluorescent image, and bottom: merged image. The $650 \mathrm{~nm}$ laser power density was $125 \mathrm{~mW} / \mathrm{cm}^{2}$.

To achieve active delivery, folic acid (FA) is one of the popular ligands used to bind cancer cells [43]. It has been reported that the number of folate-specific receptor antigens on cancer cells is higher than that for normal cells. Therefore, Au@TNA@MB NPs modified with FA molecules were prepared to evaluate the enhanced PDT treatment of T24 cells. The TEM image in Figure S7a presents the well 
dispersion for the FA-conjugated Au@TNA@MB NPs. The UV-visible spectrum of FA-conjugated Au@TNA@MB NPs determined a specific absorption pattern (Figure S7b), which is similar to the absorption band in Figure S3b, providing direct evidence for the lack of colloidal aggregation. A slight increase of the zeta potential value from $-33.7 \mathrm{mV}$ by Au@TNA@MB NPs to $-28.2 \mathrm{mV}$ by FA-conjugated $\mathrm{Au} @ \mathrm{TNA} @ \mathrm{MB}$ NPs (Figure S7c) was attributed to immobilization of the $\mathrm{NH}_{2}-\mathrm{PEG}-\mathrm{NH}_{2}$ polymer and FA molecule. Figure 5c shows that 10 ppm of FA-conjugated Au@TNA@MB NPs was capable of conducting the PDT-triggered killing of at least 50\% T24 cells at a 45 min coculture time. When the incubation time was prolonged to $4 \mathrm{~h}$, this enhanced delivery to the specific antigen of T24 cancer cells led to excellent PDT of bladder cancer cells, boosting the death rate to below $50 \%$ at $1 \mathrm{ppm}_{\text {[Au] }}$ and over $80 \%$ at $10 \mathrm{ppm}_{[\mathrm{Au}]}$. This high cancer cell mortality could be attributed to the large yield of ROS in the targeted cancer area, according to the comparison of the fluorescence intensity between the targeted PDT results (Figure 5e) and the passive delivery of the targeted-free group (Figure 5d).

Although the enhanced phototoxic effect of FA-conjugated Au@TNA@MB NPs on malignant cells is proven in Figure $5 \mathrm{c}, \mathrm{e}$, the potential side effects, e.g., injury to normal cells, remain to be considered. Regarding this, the cell viability of the human normal urinary epithelial cell line SV-HUC-1 was examined under 45-min to 4-h targeted PDT treatments with FA-conjugated Au@TNA@MB NPs. As shown in Figure 6a,b, a very low cytotoxicity for the particle-treated SV-HUC-1 cells (13\%) was observed at 10 ppm of FA-conjugated Au@TNA@MB NPs.
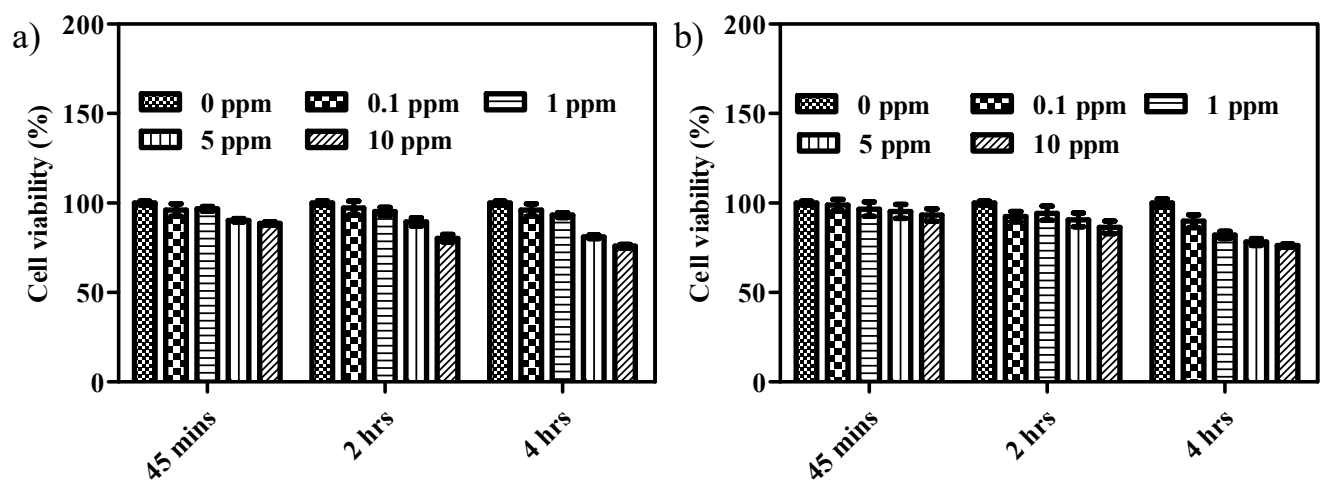

Figure 6. MTT assays of SV-HUC-1 cells cotreated with (a) Au@TNA@MB and (b) FA-conjugated Au@TNA@MB NPs for $0.75-4 \mathrm{~h}$ plus $650 \mathrm{~nm}$ light $\left(125 \mathrm{~mW} / \mathrm{cm}^{2}\right)$, followed by another $24 \mathrm{~h}$ of incubation in fresh medium.

\section{Conclusions}

We successfully synthesized Au@TNA NPs by a simple one-pot green reaction of HAuCl4 and TNA solutions without the use of toxic additives. The Au@TNA NPs could carry massive amounts of $\mathrm{MB}$ molecules featuring a high production yield of ${ }^{1} \mathrm{O}_{2}$ generation upon excitation at $650 \mathrm{~nm}$. The cytotoxicity of Au@TNA/MB NPs was very low for HeLa and T24 living cells at concentrations between 1 and 200 ppm. According to the PDT study with FA-conjugated Au@TNA/MB NPs and the T24 cell line, the target activated photocytotoxicity was better than the passive PDT effect via the endocytosis pathway. The prolonged targeted delivery time to cells could enhance the PDT-related cytotoxicity to cancer cells, but retain a very low dark toxicity to normal cells. Our in vitro study provides promising results for FA-conjugated Au@TNA/MB NPs as prospective PS nanomaterials for PDT to treat NMIBC; nevertheless, additional animal studies are required to prove the treatment efficacy toward cancer cells and to prevent adverse effects. Although it remains a big synthetic challenge to manipulate Au nanostructures with polyphenols, we expect that changing the size and shape of Au@TNA nanoparticles will enhance the affinity to cancer cells and offer a promising future design to improve the delivery efficiency of PDT NPs. 
Supplementary Materials: The following are available online at http://www.mdpi.com/2079-4991/10/7/1351/s1: Figure S1: Size distribution measurements of (a) Au@TNA NPs and (b) Au@TNA@MB NPs on the basis of TEM image measurements; Figure S2: UV-visible spectra of the MB alone and the 1st/3rd supernatant solution collected from the mixture solution of Au@TNA nanoparticle and MB molecule through a centrifugation process; Figure S3: UV-visible measurements of (a) Au@TNA NPs and (b) Au@TNA@MB NPs aged in PBS solution. Optical images of (c) Au@TNA NPs and (d) Au@TNA@MB NPs dispersed in PBS solution for 30 min; Figure S4: (a) UV-visible and (b) fluorescence measurements of the MB alone solution, referred as $100 \%$ of concentration, and the supernatant of the Au@TNA@MB nanoparticles after 1 day of aging; Figure S5: Uv-visible spectra of ABDA reacted with $200 \mu \mathrm{L}$ of (a) Au@TNA@MB nanoparticles (200 ppm[Au]) and (b) MB alone solution (0.024 mM) under an excitation at $650 \mathrm{~nm}$. The absorption changes at 400 nm of MB alone, Au@TNA NPs, and Au@TNA@MB NPs under an excitation at $650 \mathrm{~nm}$; Figure S6: UV-visible spectra of the RNO/imidazole indicator for measuring single oxygen generation from MB-alone solution as a function of the irradiation time at $650 \mathrm{~nm}\left(125 \mathrm{~mW} / \mathrm{cm}^{2}\right)$; Figure S7: (a) TEM image and (b) UV-visible spectrum of FA-conjugated Au@TNA@MB NPs. (c) Zeta potential measurements of Au@TNA, Au@TNA@MB, and FA-conjugated Au@TNA@MB NPs.

Author Contributions: Conceptualization, M.-Y.L. and Y.-C.C.; methodology, M.-Y.L.; software, T.-Y.C.; validation, N.-C.C. and T.-Y.C.; formal analysis, N.-C.C. and T.-Y.C.; data curation, M.-Y.L.; writing-original draft preparation, C.-W.H. and N.-C.C.; writing-review and editing, M.-Y.L. and Y.-C.C.; supervision, Y.-C.C.; project administration, M.-Y.L. and Y.-C.C. All authors have read and agreed to the published version of the manuscript.

Funding: This work was supported in part by grants from the Ministry of Science and Technology, Taiwan (MOST 107-2113-M-153-002-, 108-2113-M-153-001-, and 108-2622-M-006-001-CC1), and by a grant from the Taipei City Hospital (TCH) and the Department of Health, Taipei City Government (TCH No. 10801-62-001, 10901-62-001 and 10901-62-006).

Acknowledgments: We thank Yi-Jiun Chen and Chia-Jung Shen for their valuable contributions in funding coordination and management. We thank Ya-Ting Chuang and Tzu-Lan Kao for valuable help with the sample TEM and optical characterizations.

Conflicts of Interest: The authors declare no conflicts of interest.

\section{References}

1. Bray, F.; Ferlay, J.; Soerjomataram, I.; Siegel, R.L.; Torre, L.A.; Jemal, A. Global cancer statistics 2018: GLOBOCAN estimates of incidence and mortality worldwide for 36 cancers in 185 countries. Ca A Cancer J. Clin. 2018, 68, 394-424. [CrossRef]

2. Kamat, A.M.; Hahn, N.M.; Efstathiou, J.A.; Lerner, S.P.; Malmström, P.-U.; Choi, W.; Guo, C.C.; Lotan, Y.; Kassouf, W. Bladder cancer. Lancet 2016, 388, 2796-2810. [CrossRef]

3. Babjuk, M.; Böhle, A.; Burger, M.; Capoun, O.; Cohen, D.; Compérat, E.M.; Hernández, V.; Kaasinen, E.; Palou, J.; Rouprêt, M. EAU guidelines on non-muscle-invasive urothelial carcinoma of the bladder: Update 2016. Eur. Urol. 2017, 71, 447-461. [CrossRef] [PubMed]

4. Clark, P.E.; Spiess, P.E.; Agarwal, N.; Bangs, R.; Boorjian, S.A.; Buyyounouski, M.K.; Efstathiou, J.A.; Flaig, T.W.; Friedlander, T.; Greenberg, R.E. NCCN guidelines insights: Bladder cancer, version 2.2016. J. Natl. Compr. Cancer Netw. 2016, 14, 1213-1224. [CrossRef]

5. Cambier, S.; Sylvester, R.J.; Collette, L.; Gontero, P.; Brausi, M.A.; Van Andel, G.; Kirkels, W.J.; Da Silva, F.C.; Oosterlinck, W.; Prescott, S. EORTC nomograms and risk groups for predicting recurrence, progression, and disease-specific and overall survival in non-muscle-invasive stage Ta-T1 urothelial bladder cancer patients treated with 1-3 years of maintenance bacillus Calmette-Guérin. Eur. Urol. 2016, 69, 60-69. [CrossRef]

6. Prout, G.R.; Marshall, V.F. The prognosis with untreated bladder tumors. Cancer 1956, 9, 551-558. [CrossRef]

7. Thoma, C. PD-L1 and BCG response prediction. Nat. Rev. Urol. 2020, 17, 8. [CrossRef] [PubMed]

8. Agostinis, P.; Berg, K.; Cengel, K.A.; Foster, T.H.; Girotti, A.W.; Gollnick, S.O.; Hahn, S.M.; Hamblin, M.R.; Juzeniene, A.; Kessel, D. Photodynamic therapy of cancer: An update. Ca A Cancer J. Clin. 2011, 61, $250-281$. [CrossRef]

9. Konan, Y.N.; Gurny, R.; Allemann, E. State of the art in the delivery of photosensitizers for photodynamic therapy. J. Photochem. Photobiol. B Biol. 2002, 66, 89-106. [CrossRef]

10. Railkar, R.; Agarwal, P.K. Photodynamic Therapy in the Treatment of Bladder Cancer: Past Challenges and Current Innovations. Eur. Urol. Focus 2018. [CrossRef]

11. Walther, M.M. The role of photodynamic therapy in the treatment of recurrent superficial bladder cancer. Urol. Clin. N. Am. 2000, 27, 163-170. [CrossRef] 
12. Dougherty, T.J.; Gomer, C.J.; Henderson, B.W.; Jori, G.; Kessel, D.; Korbelik, M.; Moan, J.; Peng, Q. Photodynamic therapy. JNCI J. Natl. Cancer Inst. 1998, 90, 889-905. [CrossRef] [PubMed]

13. Railkar, R.; Krane, L.S.; Li, Q.Q.; Sanford, T.; Siddiqui, M.R.; Haines, D.; Vourganti, S.; Brancato, S.J.; Choyke, P.L.; Kobayashi, H. Epidermal growth factor receptor (EGFR)-targeted photoimmunotherapy (PIT) for the treatment of EGFR-expressing bladder cancer. Mol. Cancer Ther. 2017, 16, 2201-2214. [CrossRef] [PubMed]

14. Yong, K.-T.; Law, W.-C.; Hu, R.; Ye, L.; Liu, L.; Swihart, M.T.; Prasad, P.N. Nanotoxicity assessment of quantum dots: From cellular to primate studies. Chem. Soc. Rev. 2013, 42, 1236-1250. [CrossRef]

15. Wang, L.-S.; Chuang, M.-C.; Ho, J.A.A. Nanotheranostics-A review of recent publications. Int. J. Nanomed. 2012, 7, 4679 .

16. Jain, S.; Hirst, D.; O'sullivan, J. Gold nanoparticles as novel agents for cancer therapy. Br. J. Radiol. 2012, 85, 101-113. [CrossRef]

17. Hong, W.-E.; Hsu, I.-L.; Huang, S.-Y.; Lee, C.-W.; Ko, H.; Tsai, P.-J.; Shieh, D.-B.; Huang, C.-C. Assembled growth of $3 \mathrm{D} \mathrm{Fe}_{3} \mathrm{O}_{4}{ }^{\circledR} \mathrm{Au}$ nanoparticles for efficient photothermal ablation and SERS detection of microorganisms. J. Mater. Chem. B 2018, 6, 5689-5697. [CrossRef]

18. Tai, Y.-W.; Chiu, Y.-C.; Wu, P.-T.; Yu, J.; Chin, Y.-C.; Wu, S.-P.; Chuang, Y.-C.; Hsieh, H.-C.; Lai, P.-S.; Yu, H.-P. Degradable nir-ptt nanoagents with a potential $\mathrm{Cu}^{@} \mathrm{Cu}_{2} \mathrm{O}^{@}$ polymer structure. ACS Appl. Mater. Interfaces 2018, 10, 5161-5174. [CrossRef]

19. Burdușel, A.-C.; Gherasim, O.; Grumezescu, A.M.; Mogoantă, L.; Ficai, A.; Andronescu, E. Biomedical applications of silver nanoparticles: An up-to-date overview. Nanomaterials 2018, 8, 681. [CrossRef]

20. Xiao, H.; Li, C.; Dai, Y.; Cheng, Z.; Hou, Z.; Lin, J. Inorganic nanocarriers for platinum drug delivery. Mater. Today 2015, 18, 554-564.

21. Redmond, R.W.; Gamlin, J.N. A compilation of singlet oxygen yields from biologically relevant molecules. Photochem. Photobiol. 1999, 70, 391-475. [CrossRef]

22. Tardivo, J.P.; Del Giglio, A.; De Oliveira, C.S.; Gabrielli, D.S.; Junqueira, H.C.; Tada, D.B.; Severino, D.; De Fátima Turchiello, R.; Baptista, M.S. Methylene blue in photodynamic therapy: From basic mechanisms to clinical applications. Photodiagn. Photodyn. Ther. 2005, 2, 175-191. [CrossRef]

23. Deng, T.; Li, J.S.; Jiang, J.H.; Shen, G.L.; Yu, R.Q. Preparation of Near-IR Fluorescent Nanoparticles for Fluorescence-Anisotropy-Based Immunoagglutination Assay in Whole Blood. Adv. Funct. Mater. 2006, 16, 2147-2155. [CrossRef]

24. He, X.; Wu, X.; Wang, K.; Shi, B.; Hai, L. Methylene blue-encapsulated phosphonate-terminated silica nanoparticles for simultaneous in vivo imaging and photodynamic therapy. Biomaterials 2009, 30, 5601-5609. [CrossRef] [PubMed]

25. Liu, T.-M.; Conde, J.; Lipiński, T.; Bednarkiewicz, A.; Huang, C.-C. Smart NIR linear and nonlinear optical nanomaterials for cancer theranostics: Prospects in photomedicine. Prog. Mater. Sci. 2017, 88, 89-135. [CrossRef]

26. Zhou, W.; Gao, X.; Liu, D.; Chen, X. Gold nanoparticles for in vitro diagnostics. Chem. Rev. 2015, 115, 10575-10636. [CrossRef]

27. Li, N.; Zhao, P.; Astruc, D. Anisotropic gold nanoparticles: Synthesis, properties, applications, and toxicity. Angew. Chem. Int. Ed. 2014, 53, 1756-1789. [CrossRef]

28. Conde, J.; Dias, J.T.; Grazú, V.; Moros, M.; Baptista, P.V.; De la Fuente, J.M. Revisiting 30 years of biofunctionalization and surface chemistry of inorganic nanoparticles for nanomedicine. Front. Chem. 2014, 2, 48. [CrossRef]

29. Calavia, P.G.; Bruce, G.; Pérez-García, L.; Russell, D.A. Photosensitiser-gold nanoparticle conjugates for photodynamic therapy of cancer. Photochem. Photobiol. Sci. 2018, 17, 1534-1552. [CrossRef]

30. Lin, J.; Wang, S.; Huang, P.; Wang, Z.; Chen, S.; Niu, G.; Li, W.; He, J.; Cui, D.; Lu, G. Photosensitizer-loaded gold vesicles with strong plasmonic coupling effect for imaging-guided photothermal/photodynamic therapy. ACS Nano 2013, 7, 5320-5329. [CrossRef]

31. Boruah, S.K.; Boruah, P.K.; Sarma, P.; Medhi, C.; Medhi, O.K. Green synthesis of gold nanoparticles using Camellia sinensis and kinetics of the reaction. Adv. Mat. Lett. 2012, 3, 481-486. [CrossRef]

32. Lee, C.-W.; Ko, H.; Chang, S.-H.G.; Huang, C.-C. Invisible-ink-assisted pattern and written surface-enhanced Raman scattering substrates for versatile chem/biosensing platforms. Green Chem. 2018, 20, 5318-5326. [CrossRef] 
33. Chitgupi, U.; Qin, Y.; Lovell, J.F. Targeted nanomaterials for phototherapy. Nanotheranostics 2017, 1, 38. [CrossRef] [PubMed]

34. Yang, Y.-T.; Hsu, I.-L.; Cheng, T.-Y.; Wu, W.-J.; Lee, C.-W.; Li, T.-J.; Cheung, C.I.; Chin, Y.-C.; Chen, H.-C.; Chiu, Y.-C. Off-Resonance SERS Nanoprobe-Targeted Screen of Biomarkers for Antigens Recognition of Bladder Normal and Aggressive Cancer Cells. Anal. Chem. 2019, 91, 8213-8220. [CrossRef] [PubMed]

35. Zhang, Q.; Xie, J.; Yang, J.; Lee, J.Y. Monodisperse icosahedral Ag, Au, and Pd nanoparticles: Size control strategy and superlattice formation. ACS Nano 2009, 3, 139-148. [CrossRef] [PubMed]

36. Vericat, C.; Vela, M.; Benitez, G.; Carro, P.; Salvarezza, R. Self-assembled monolayers of thiols and dithiols on gold: New challenges for a well-known system. Chem. Soc. Rev. 2010, 39, 1805-1834. [CrossRef]

37. Song, X.; Gong, H.; Liu, T.; Cheng, L.; Wang, C.; Sun, X.; Liang, C.; Liu, Z. J-Aggregates of organic dye molecules complexed with iron oxide nanoparticles for imaging-guided photothermal therapy under 915-nm light. Small 2014, 10, 4362-4370. [CrossRef]

38. Liu, R.; Zhao, J.; Han, G.; Zhao, T.; Zhang, R.; Liu, B.; Liu, Z.; Zhang, C.; Yang, L.; Zhang, Z. Click-functionalized SERS Nanoprobes with improved labeling efficiency and capability for cancer cell imaging. ACS Appl. Mater. Interfaces 2017, 9, 38222-38229. [CrossRef]

39. Yi, X.; Dai, J.; Han, Y.; Xu, M.; Zhang, X.; Zhen, S.; Zhao, Z.; Lou, X.; Xia, F. A high therapeutic efficacy of polymeric prodrug nano-assembly for a combination of photodynamic therapy and chemotherapy. Commun. Biol. 2018, 1, 1-13. [CrossRef]

40. Acedo, P.; Stockert, J.; Cañete, M.; Villanueva, A. Two combined photosensitizers: A goal for more effective photodynamic therapy of cancer. Cell Death Dis. 2014, 5, e1122. [CrossRef]

41. Van Rixel, V.; Siewert, B.; Hopkins, S.; Askes, S.; Busemann, A.; Siegler, M.; Bonnet, S. Green light-induced apoptosis in cancer cells by a tetrapyridyl ruthenium prodrug offering two trans coordination sites. Chem. Sci. 2016, 7, 4922-4929. [CrossRef] [PubMed]

42. Elmore, S. Apoptosis: A review of programmed cell death. Toxicol. Pathol. 2007, 35, 495-516. [CrossRef] [PubMed]

43. Pan, D.; Turner, J.L.; Wooley, K.L. Folic acid-conjugated nanostructured materials designed for cancer cell targeting. Chem. Commun. 2003, 19, 2400-2401. [CrossRef] [PubMed]

(C) 2020 by the authors. Licensee MDPI, Basel, Switzerland. This article is an open access article distributed under the terms and conditions of the Creative Commons Attribution (CC BY) license (http://creativecommons.org/licenses/by/4.0/). 